\title{
Cosine families and semigroups really differ
}

\author{
ADAM BOBROWSKI AND WOJCIECH CHOJNACKI
}

\section{Dedicated to Professor Jan Kisyński on the occasion of his 80th birthday}

Abstract. We reveal three surprising properties of cosine families, distinguishing them from semigroups of operators: (1) A single trajectory of a cosine family is either strongly continuous or not measurable. (2) Pointwise convergence of a sequence of equibounded cosine families implies that the convergence is almost uniform for time in the entire real line; in particular, cosine families cannot be perturbed in a singular way. (3) A non-constant trajectory of a bounded cosine family does not have a limit at infinity; in particular, the rich theory of asymptotic behaviour of semigroups does not have a counterpart for cosine families. In addition, we show that equibounded cosine families that converge strongly and almost uniformly in time may fail to converge uniformly.

\section{Introduction}

The theory of cosine families is in many aspects parallel to the theory of semigroups of operators $[1,20]$. For example, the fundamental theorem of Sova-DaPratoGiusti on the generation of cosine families is akin to the Hille-Yosida-Feller-PhillipsMiyadera theorem on the generation of semigroups, and both results have in fact a common source in the Henning-Neubrander representation theorem [1,4]. Similarly, the classical form of the Trotter-Kato-Neveu theorem on the convergence of semigroups carries over to cosine families with only minor changes [4, 19,20,25]. However, despite similarities like those above, there are also fundamental differences between the theory of cosine families and that of semigroups. This paper will expose a number of differences between cosine families and semigroups which in our opinion are of fundamental nature.

The first of the differences that we shall reveal is embedded into a wider context of operator-valued functional equations. Functional equations satisfied by families of bounded linear operators related to Cauchy problems imply that the trajectories of the families exhibit surprising automatic continuity properties. A representative example

Mathematics Subject Classification (2010): Primary 47D09, 34G10; Secondary 60J65

Keywords: Cosine family, semigroup of operators, strong measurability, strong continuity, convergence.

Adam Bobrowski is on leave from Department of Mathematics, Faculty of Electrical Engineering and Computer Science, Lublin University of Technology, Nadbystrzycka 38A, 20-618 Lublin, Poland. 
here is the classic result due to Dunford, Hille, and Phillips [22, p. 305], asserting that a strongly measurable semigroup on a Banach space is strongly continuous on $(0, \infty)$. A semigroup which is strongly continuous on $(0, \infty)$ may fail to be continuous at the origin, and this justifies the need for dividing semigroups into classes-such as Abel summable and strongly continuous semigroups - according to their regularity at the origin. A counterpart of the Dunford-Hille-Phillips result for cosine families is the theorem of Fattorini (see [16, Lemma 5.2] and [18, pp. 24-28]) stating that a strongly measurable cosine family on a Banach space is strongly continuous on $\mathbb{R}$.

Referring to Fattorini's theorem, some authors (see e.g. [28, p. 529] and [29, Proposition 2.1.2]) cite-somewhat confusingly - a weaker and later result, namely the theorem of Chander and Singh [13] (see also [26]) asserting that a strongly measurable cosine family on a Banach space is strongly continuous on $\mathbb{R} \backslash\{0\}$. But this is not a grave issue. Indeed, we show in Sect. 3, as the first result of this paper, that Fattorini's theorem can easily be deduced from Chander and Singh's. Following this, we show-in the same section-that Fattorini's theorem can be further extended, namely we prove that a single trajectory of a cosine family is either continuous on $\mathbb{R}$, or not measurable. Fattorini's and our result together explain why there is no need for a cosine family analogue of Abel summable and related classes of semigroups - all measurable cosine families form just one class-and also mark the first fundamental difference between the theories of cosine families and semigroups.

Fattorini's theorem and our refinement of it fit well with the theorem of Hille [16, $17,20,21]$ saying that for $n \geq 3$ the $n$th order Cauchy problem is well posed if and only if the generating operator of the problem is bounded: the solution families for the higher-order Cauchy problems are uniformly continuous. In other words, the stronger character of the automatic continuity results compared to the Dunford-Hille-Phillips result is in line with the fact that the regularity of solutions of a Cauchy problem increases with the order of the problem.

We exhibit further differences between cosine families and semigroups in Sect. 4 where we consider the question of convergence of sequences of cosine families and semigroups. (In fact, this question was the main motivation for our study and led us to consider the problem of automatic continuity of trajectories of cosine families.) To explain our starting point, we recall that according to the classical form of the TrotterKato theorem if, given a sequence of equibounded strongly continuous semigroups on a Banach space $X$, the resolvents of the generators of the semigroups converge strongly and the range $\mathscr{R}$ of the limit pseudo-resolvent is dense in $X$, then the semigroups converge strongly and almost uniformly on $[0, \infty)[1,6,15,20]$. If $\mathscr{R}$ is not dense in $X$, then, in agreement with the extended form of the Trotter-Kato theorem, the semigroups converge almost uniformly on $[0, \infty)$ merely on the closure of $\mathscr{R}$, this space being commonly referred to as the regularity space. However, especially in the context of singular perturbations, it may happen that the semigroups do converge outside the regularity space-the convergence is then almost uniform on $(0, \infty)$ and 
cannot be deduced from the sole convergence of resolvents; see e.g. [2,3,5,7-9,11] and references given therein.

Strikingly, examples of such an irregular behaviour have not been observed for cosine families. To discuss this in more detail, we first note that the Trotter-Kato theorem in extended form also holds for cosine families, with the notion of a regularity space playing a similar role as for semigroups [4]. We now point out that in [4] and [10] sequences of equibounded cosine families were constructed such that (i) the regularity space is a proper subset of $X$, (ii) the associated semigroups given by the abstract Weierstrass formula converge also outside the regularity space, and (iii) the cosine families do not converge outside the regularity space. These examples suggest that cosine families by nature cannot converge in an irregular manner like that described above with regard to semigroups. We give a precise statement of this assertion in the main result of Sect. 4. The corresponding proof will draw on Fattorini's theorem mentioned earlier. One noteworthy consequence of our result is that there is no singular perturbation theory for cosine families analogous to that for semigroups of operators.

As a final contribution in Sect. 3, we show that no non-constant trajectory of a bounded cosine family has a limit at infinity. This result, generalising [1, Proposition 3.14.6], has the consequence that the important theory of asymptotic behaviour of semigroups $[15,27]$ does not have a cosine family analogue.

The examples presented in [4] and [10] have led to the conjecture that if equibounded cosine families converge strongly, they do so uniformly on $\mathbb{R}$, and not just almost uniformly. If true, the message conveyed in this conjecture would mark another difference between semigroups and cosine families. However, as we show in the final Sect. 5, the conjecture does not hold-equibounded cosine families like equibounded semigroups may fail to converge uniformly. In particular, we give several examples of cosine families, each of which is a common strong limit of two sequences of cosine families, of which one converges uniformly and the other converges merely almost uniformly on $\mathbb{R}$.

\section{Preliminaries}

We first review some of the concepts and terminology used in the paper.

Let $X$ be a Banach space. We denote by $\mathscr{L}(X)$ the space of all bounded linear operators on $X$ and by $I_{X}$ the identity operator on $X$. An $\mathscr{L}(X)$-valued family is said to be a cosine family on $X$ if

(i) $2 C(t) C(s)=C(t+s)+C(t-s)$ for all $s, t \in \mathbb{R}$ (d'Alembert's functional equation, also called the cosine functional equation),

(ii) $C(0)=I_{X}$.

The concept of a cosine family on a Banach space is closely related to the more familiar concept of a one-parameter semigroup of operators on a Banach space. A family $\{S(t)\}_{t \geq 0}$ of bounded linear operators on a Banach space $X$ is a semigroup of operators on $X$ if 
(i) $S(s) S(t)=S(s+t)$ for all $s, t \geq 0$ (Cauchy's equation, also called the exponential equation),

(ii) $S(0)=I_{X}$.

Our main focus throughout the paper will be on strongly continuous cosine families and semigroups on a Banach space. We recall that a family $F=\{F(t)\}_{t \in T}$ of bounded linear operators on a Banach space $X$ indexed by $T \subset \mathbb{R}$ is strongly continuous if, for each $x \in X$, the $F$-trajectory (or simply trajectory) associated with $x, T \ni t \mapsto$ $F(t) x \in X$, is continuous in norm. We also recall that strongly continuous semigroups and cosine families on a Banach space are uniquely characterised by their respective generators. The generator $A$ of a strongly continuous semigroup $\{S(t)\}_{t \geq 0}$ on a Banach space $X$ is defined by

$$
A x=\left.\frac{\mathrm{d}}{\mathrm{d} t}\right|_{0} S(t) x=\lim _{s \rightarrow 0} \frac{S(s) x-x}{s} \quad(x \in D(A)),
$$

where $D(A)$, the domain of $A$, is the set of all $x \in X$ for which the derivative (2.1) exists. In turn, the generator $A$ of a strongly continuous cosine family $\{C(t)\}_{t \in \mathbb{R}}$ on $X$ is defined by

$$
A x=\left.\frac{\mathrm{d}^{2}}{\mathrm{~d} t^{2}}\right|_{0} C(t) x=\lim _{s \rightarrow 0} \frac{2}{s^{2}}(C(s) x-x) \quad(x \in D(A)),
$$

where $D(A)$ is the set of all $x \in X$ for which the second derivative (2.2) exists. For standard results concerning strongly continuous semigroups and cosine families and their corresponding generators, the reader is referred to, e.g. [1,18], or [20].

Some of the cosine families and semigroups that we shall consider will be bounded. We recall that a family $F=\{F(t)\}_{t \in T}$ of bounded linear operators on a Banach space $X$ indexed by $T \subset \mathbb{R}$ is bounded if $\sup _{t \in T}\|F(t)\|<\infty$.

We shall also make use of the concept of a strongly measurable family of operators on a Banach space. A family $\{F(t)\}_{t \in T}$ of linear bounded operators on a Banach space $X$ indexed by a measurable set $T \subset \mathbb{R}$ is strongly measurable if, for each $x \in X$, the function $t \mapsto F(t) x$ is Bochner measurable on $T$.

\section{Measurability implies continuity}

The following theorem of Fattorini, already mentioned in the Introduction, is fundamental to subsequent considerations, having a direct bearing on the issue of convergence of cosine families which is of concern in this paper.

THEOREM 1 (Fattorini). A strongly measurable cosine family on a Banach space is strongly continuous on $\mathbb{R}$.

As it turns out, Fattorini's theorem is an immediate consequence of the seemingly weaker, but perhaps better known theorem of Chander and Singh, also already mentioned in the Introduction, stating that a strongly measurable cosine family on a Banach 
space is strongly continuous on $\mathbb{R} \backslash\{0\}$. The link between the two theorems is provided by the following result:

PROPOSITION 1. Let $\{C(t)\}_{t \in \mathbb{R}}$ be a cosine family on a Banach space X. Let $x \in X$ be such that the function $t \mapsto C(t) x$ is continuous on $(a, \infty)$ for some $a \in \mathbb{R}$. Then the function $t \mapsto C(t) x$ is continuous on $\mathbb{R}$.

Proof. Fix $t \in \mathbb{R}$ arbitrarily. Choose $h>0$ so that $t+h>a+1$. We clearly have

$$
C(\tau) x=2 C(h) C(\tau+h) x-C(\tau+2 h) x
$$

for each $\tau \in \mathbb{R}$. Noting that $\tau+h>a$ and $\tau+2 h>a$ whenever $\tau>t-1$ and exploiting the assumption, we get

$$
\lim _{\tau \rightarrow t} C(\tau+h) x=C(t+h) x \text { and } \lim _{\tau \rightarrow t} C(\tau+2 h) x=C(t+2 h) x .
$$

As $C(h)$ is a bounded operator, we also have

$$
\lim _{\tau \rightarrow t} C(h) C(\tau+h) x=C(h) C(t+h) x .
$$

Therefore,

$$
\lim _{\tau \rightarrow t} C(\tau) x=2 C(h) C(t+h) x-C(t+2 h) x=C(t) x .
$$

Since $t$ was chosen arbitrarily, the result follows.

We remark that the above proposition has analogues for solutions of other functional equations (see e.g. the proof of [28, Thm. 2.1]), and these can in turn be used to establish suitable counterparts of Theorem 1.

Fattorini's theorem has a global flavour, in that it concerns the set of all trajectories of a cosine family. Below, we establish a local version of Fattorini's result pertaining to any individual trajectory.

THEOREM 2. Let $\{C(t)\}_{t \in \mathbb{R}}$ be a cosine family on a Banach space $X$. Let $x \in X$ be such that the function $t \mapsto C(t) x$ is measurable on $\mathbb{R}$. Then the function $t \mapsto C(t) x$ is continuous on $\mathbb{R}$.

Proof. Let $X_{0}$ be the set of all those $y \in X$ for which $t \mapsto C(t) y$ is measurable on $\mathbb{R}$. It is clear that $X_{0}$ is a linear subspace of $X$. Since the pointwise limit of a sequence of measurable functions is a measurable function, we see that $X_{0}$ is closed. Moreover, $X_{0}$ is invariant for each operator $C(s), s \in \mathbb{R}$; for if $s \in \mathbb{R}$ and $y \in X_{0}$, then $t \mapsto C(t+s) y$ and $t \mapsto C(t-s) y$ are both measurable, and consequently, $t \mapsto C(t) C(s) y=(C(t+s) x+C(t-s) y) / 2$ is measurable, too. Now, if we consider the cosine family $\left\{C(t)_{\mid X_{0}}\right\}_{t \in \mathbb{R}}$ on $X_{0}$, where $C(t)_{\mid X_{0}}$ denotes the restriction of $C(t)$ to $X_{0}$, then it is clear that this family is strongly measurable, and so, by Theorem 1 , it is in fact strongly continuous. Since $x$ is in $X_{0}$, it follows that $t \mapsto C(t) x$ is continuous, and the theorem is proved. 


\section{Convergence is regular}

The Trotter-Kato theorem for cosine families, pertaining to the case where cosine families converge to a cosine family strongly and uniformly on compact time intervals, is completely analogous to the Trotter-Kato theorem for semigroups of operators (cf. [20, §8.6]; see also [19] and [25]). Based on this analogy, one might think that generally if cosine families converge, they do so in a fashion similar to that exhibited by semigroups. However, as it turns out, there is a fundamental difference in the behaviour of semigroups and cosine families with regard to convergence which reveals itself only when one considers situations more general than that captured by the Trotter-Kato theorem. Here, we expose this difference and show that the convergence of cosine families is as a rule far more regular than that of semigroups.

We start by recalling the scenario that holds for semigroups. For each $n \in \mathbb{N}$, let $A_{n}$ generate a strongly continuous semigroup $S_{n}=\left\{S_{n}(t)\right\}_{t \geq 0}$ on a Banach space $X$. Assume that the semigroups $S_{n}$ are equibounded, i.e. there is a constant $M>0$ such that

$$
\sup _{n \in \mathbb{N}, t \geq 0}\left\|S_{n}(t)\right\| \leq M
$$

and that, for each $\lambda>0$, the resolvents of the generators $A_{n}$,

$$
\left(\lambda-A_{n}\right)^{-1}=\int_{0}^{\infty} \mathrm{e}^{-\lambda t} S_{n}(t) \mathrm{d} t,
$$

converge to an operator $R_{\lambda}$ in the strong operator topology. The $R_{\lambda}$ 's then form a pseudo-resolvent - a family of operators satisfying the first resolvent equation, with a common null space and a common range. Note that, if the $S_{n}$ 's converge strongly (on all of $X)$, then the convergence of the $\left(\lambda-A_{n}\right)^{-1}$ is a consequence of the representation (4.1) and the Lebesgue dominated convergence theorem. Associated with the $S_{n}$ 's are two subspaces of $X$ :

$$
\begin{aligned}
X_{\text {point }}^{\mathrm{s}}= & \left\{x \in X \mid \lim _{n \rightarrow \infty} S_{n}(t) x \text { exists for each } t \in[0, \infty)\right\}, \\
X_{\text {almost-unif }}^{\mathrm{s}}= & \left\{x \in X \mid \lim _{n \rightarrow \infty} S_{n}(t) x \text { exists for each } t \in[0, \infty)\right. \\
& \text { and is almost uniform in } t \in[0, \infty)\},
\end{aligned}
$$

where the superscript "s" is reminiscent of the term "semigroup." In other words, $X_{\text {point }}^{\mathrm{s}}$ consists of vectors for which the corresponding trajectories of the semigroups converge pointwise, and $X_{\text {almost-unif }}^{\mathrm{s}}$ consists of vectors for which the corresponding trajectories of the semigroups converge almost uniformly on $[0, \infty)$. Clearly,

$$
X_{\text {almost-unif }}^{\mathrm{s}} \subset X_{\text {point }}^{\mathrm{s}} .
$$

As it turns out, $X_{\text {almost-unif }}^{\mathrm{s}}$ coincides with the regularity space of the pseudo-resolvent $\left\{R_{\lambda}\right\}_{\lambda>0}$, which is defined as the closure of the common range $\mathscr{R}$ of the $R_{\lambda}$ 's:

$$
X_{\text {almost-unif }}^{\mathrm{s}}=\overline{\mathscr{R}}
$$


with the bar denoting the set closure. Moreover, if the set $X_{\text {point }}^{\mathrm{s}} \backslash X_{\text {almost-unif }}^{\mathrm{s}}$ is nonempty, then it consists of vectors for which the corresponding trajectories of the semigroups converge almost uniformly in $t \in(0, \infty)$, but not uniformly on any compact interval in $[0, \infty)$ containing 0 ; see [5]. Of course, for vectors outside $X_{\text {point }}^{\mathrm{s}}$, the corresponding trajectories of the semigroups do not converge at all. In the situation treated in the classical version of the Trotter-Kato theorem, we have $\overline{\mathscr{R}}=X$ and then $X_{\text {almost-unif }}^{\mathrm{s}}=X_{\text {point }}^{\mathrm{s}}=X$, which is a rather special case. In general, $X_{\text {almost-unif }}^{\mathrm{s}}$ is a proper subspace of $X_{\text {point }}^{\mathrm{s}}$, and $X_{\text {point }}^{\mathrm{s}}$ is a proper subspace of $X$.

For example, in the space $\mathbb{C}^{3}$, the semigroups

$$
S_{n}(t)\left(\begin{array}{l}
x \\
y \\
z
\end{array}\right)=\left(\begin{array}{c}
\mathrm{e}^{-a_{n} t} x \\
\mathrm{e}^{-n t} y \\
\mathrm{e}^{i t n} z
\end{array}\right)
$$

generated by

$$
A_{n}\left(\begin{array}{l}
x \\
y \\
z
\end{array}\right)=\left(\begin{array}{c}
-a_{n} x \\
-n y \\
i n z
\end{array}\right)
$$

where $\left(a_{n}\right)_{n \geq 1}$ is a sequence of non-negative numbers converging to $a \geq 0$, are equibounded with bound $M=1$, and the corresponding resolvents of the $A_{n}$ 's converge:

$$
\lim _{n \rightarrow \infty}\left(\lambda-A_{n}\right)^{-1}\left(\begin{array}{l}
x \\
y \\
z
\end{array}\right)=\lim _{n \rightarrow \infty}\left(\begin{array}{c}
\frac{x}{\lambda+a_{n}} \\
\frac{y}{\lambda+n} \\
\frac{z}{\lambda-i n}
\end{array}\right)=\left(\begin{array}{c}
\frac{x}{\lambda+a} \\
0 \\
0
\end{array}\right) \quad(\lambda>0) .
$$

In this case, $X_{\text {almost-unif }}^{\mathrm{s}}=\mathbb{C} \times\{0\} \times\{0\}$ and $X_{\text {point }}^{\mathrm{s}}=\mathbb{C} \times \mathbb{C} \times\{0\}$. For more involved examples, see e.g. $[2,3,5,7-9,11]$ and the references given there.

It turns out that the above picture changes when semigroups are replaced by cosine families: with the notation analogous to the one employed above and fully explained below, we have

$$
X_{\text {point }}^{\mathrm{c}}=X_{\text {almost-unif }}^{\mathrm{c}} \text {. }
$$

In other words, the trajectories of equibounded cosine families either converge almost uniformly in $t \in \mathbb{R}$ or do not converge at all. This dichotomy is the main point of discussion in this section.

Proceeding to details, let $\left(C_{n}\right)_{n \in \mathbb{N}}$ be a sequence of strongly continuous equibounded cosine families on $X$ - that is, such that $\sup _{n \in \mathbb{N}, t \in \mathbb{R}}\left\|C_{n}(t)\right\|<\infty$. Let $\left(A_{n}\right)_{n \in \mathbb{N}}$ be the sequence of the corresponding generators. Furthermore, suppose that the resolvents of the $A_{n}$ 's,

$$
\left(\lambda-A_{n}\right)^{-1}=\lambda^{-1 / 2} \int_{0}^{\infty} \mathrm{e}^{-\sqrt{\lambda} t} C_{n}(t) \mathrm{d} t \quad(\lambda>0),
$$


converge strongly to a pseudo-resolvent $\left\{R_{\lambda}\right\}_{\lambda>0}$. Again, we remark that the strong convergence of the $\left(\lambda-A_{n}\right)^{-1}$,s is a necessary condition for the strong convergence of the $C_{n}$ 's. Let

$$
\begin{aligned}
X_{\text {point }}^{\mathrm{c}}= & \left\{x \in X \mid \lim _{n \rightarrow \infty} C_{n}(t) x \text { exists for each } t \in \mathbb{R}\right\}, \\
X_{\text {almost-unif }}^{\mathrm{c}}= & \left\{x \in X \mid \lim _{n \rightarrow \infty} C_{n}(t) x \text { exists for each } t \in \mathbb{R}\right. \\
& \text { and is almost uniform in } t \in \mathbb{R}\} \\
= & \left\{x \in X \mid \lim _{n \rightarrow \infty} C_{n}(t) x \text { exists for each } t \in[0, \infty)\right. \\
& \text { and is almost uniform in } t \in[0, \infty)\} .
\end{aligned}
$$

Then, clearly,

$$
X_{\text {almost-unif }}^{\mathrm{c}} \subset X_{\text {point }}^{\mathrm{c}} .
$$

We also have, in analogy to (4.2),

$$
X_{\text {almost-unif }}^{\mathrm{c}}=\overline{\mathscr{R}},
$$

as we show next. We start by pointing out that the inclusion $\overline{\mathscr{R}} \subset X_{\text {almost-unif }}^{\mathrm{c}}$ has already been established in [4]. To prove the reverse inclusion, for each $n \in \mathbb{N}$, let $S_{n}=\left\{S_{n}(t)\right\}_{t \geq 0}$ be the strongly continuous semigroup on $X$ related to $C_{n}$ by the abstract Weierstrass formula

$$
S_{n}(t) x=\frac{1}{\sqrt{\pi t}} \int_{0}^{\infty} \mathrm{e}^{-\tau^{2} / 4 t} C_{n}(\tau) x \mathrm{~d} \tau \quad(t>0, x \in X) .
$$

Then, as is well known, the generator of $S_{n}$ coincides with $A_{n}$ (see e.g. [1, proof of Theorem 3.14.17] or [20, Theorem 8.7]). Now, if $x$ is in $X_{\text {almost-unif }}^{\mathrm{c}}$ so that $\lim _{n \rightarrow \infty} C_{n}(t) x$ exists for all $t \in[0, \infty)$ and is almost uniform in $t \in[0, \infty)$, then also $\lim _{n \rightarrow \infty} S_{n}(t) x$ exists for all $t \in[0, \infty)$ and is almost uniform in $t \in[0, \infty)$, meaning that $x$ is a member of the space $X_{\text {almost-unif }}^{\mathrm{s}}$ associated with the $S_{n}$ 's. But then, by (4.2), $x$ is automatically a member of $\overline{\mathscr{R}}$, proving that $X_{\text {almost-unif }}^{\mathrm{c}} \subset \overline{\mathscr{R}}$.

With (4.6) established, we are ready for the main result of this section.

THEOREM 3. For equibounded, strongly continuous cosine families $C_{n}=$ $\left\{C_{n}(t)\right\}_{t \in \mathbb{R}}, n \in \mathbb{N}$, on a Banach space $X$, under the assumption of strong convergence of the resolvents of the generators of the $C_{n}$ 's, we have

$$
X_{\text {point }}^{\mathrm{c}}=X_{\text {almost-unif }}^{\mathrm{c}} \text {. }
$$

Proof. We only need to show that $X_{\text {point }}^{\mathrm{c}} \subset X_{\text {almost-unif }}^{\mathrm{c}}$ One possible proof of this inclusion involves an argument similar to the one that leads to [5, Propositions 1 and 2]. Below, however, we shall present a much simpler proof based on (4.6).

In view of the equiboundedness of the $C_{n}$ 's, $X_{\text {point }}^{\mathrm{c}}$ is a closed subspace of $X$. For each $t \in \mathbb{R}$, define the bounded operator $C(t): X_{\text {point }}^{\mathrm{c}} \rightarrow X$ by

$$
C(t) x:=\lim _{n \rightarrow \infty} C_{n}(t) x \quad\left(x \in X_{\text {point }}^{\mathrm{c}}\right) .
$$


Invoking the equiboundedness of the $C_{n}$ 's again and taking into account that each $C_{n}$ satisfies the cosine functional equation, we deduce that for each $t \in \mathbb{R}, C(t)$ maps $X_{\text {point }}^{\mathrm{c}}$ into itself and $C=\{C(t)\}_{t \in \mathbb{R}}$ satisfies the cosine functional equation. Thus, $C$ is a cosine family on $X_{\text {point }}^{\mathrm{c}}$. Being the strong limit of $\left(C_{n}\right)_{n \in \mathbb{N}}, C$ is strongly measurable. By Theorem 1, $C$ is in fact strongly continuous in $t \in \mathbb{R}$. Let $A$ be the generator of $C$. By the hypothesis, the definition of $C$, the representation (4.5), and its analogue for $A$, and the Lebesgue dominated convergence theorem, we have

$$
R_{\lambda} x=\lim _{n \rightarrow \infty}\left(\lambda-A_{n}\right)^{-1} x=(\lambda-A)^{-1} x
$$

whenever $x \in X_{\text {point }}^{\mathrm{c}}$ and $\lambda>0$; here, of course, $\left\{R_{\lambda}\right\}_{\lambda>0}$ is the limit pseudo-resolvent, defined on all of $X$, corresponding to the resolvents of the generators $A_{n}$ of the $C_{n}$ 's. Now, if $x$ is in the domain $D(A)$ of $A$, then $x=(\lambda-A)^{-1} y$, where $y=(\lambda-A) x \in$ $X_{\text {point }}^{\mathrm{c}}$, and hence also $x=R_{\lambda} y$. This means that $D(A) \subset \mathscr{R}$. Invoking (4.6), we infer that $D(A) \subset X_{\text {almost-unif }}^{\mathrm{c}}$. Since $D(A)$ is dense in $X_{\text {point }}^{\mathrm{c}}$ and both $X_{\text {point }}^{\mathrm{c}}$ and $X_{\text {almost-unif }}^{\mathrm{c}}$ are closed, it follows that $X_{\text {point }}^{\mathrm{c}} \subset X_{\text {almost-unif }}^{\mathrm{c}}$. The proof is now complete.

The above theorem may be phrased as follows: outside the regularity space, equibounded cosine families always fail to converge strongly. In light of our earlier discussion, an analogous statement for semigroups of operators is not true. We illustrate the situation with an example which is representative of the examples mentioned in the Introduction that served as original motivation behind Theorem 3 . Let $\left(\epsilon_{n}\right)_{n \in \mathbb{N}}$ be a sequence of positive numbers converging to zero. In $X=C[0, \infty]$, the space of continuous functions on $[0, \infty)$ with a limit at infinity, the operators $A_{n} f=\frac{1}{2} f^{\prime \prime}$ with domains $D\left(A_{n}\right)$, composed of twice continuously differentiable functions $f$ in $X$ with $f^{\prime \prime}$ in $X$ such that $f(0)=\epsilon_{n} f^{\prime}(0)$ generate cosine families that are bounded in norm by 3 . The resolvents of the $A_{n}$ 's converge strongly, and the closure of the range of the limit pseudo-resolvent is the subspace $C_{0}(0, \infty]$ of all those functions in $C[0, \infty]$ that vanish at 0 . In other words, the regularity space here is $C_{0}(0, \infty]$. We remark in passing that the semigroups generated by the $A_{n}$ 's are related to elastic Brownian motions $[6,23,24]$, and the limit semigroup on $C_{0}(0, \infty]$ is related to the minimal Brownian motion. As it turns out, the semigroups generated by the $A_{n}$ 's do converge strongly outside $C_{0}(0, \infty)$, with the convergence being almost uniform in $t>0[3,5]$. However, as was shown in [10], the cosine families generated by the $A_{n}$ 's do not converge strongly outside $C_{0}(0, \infty)$. Theorem 3 makes it clear that this lack of convergence is not an accidental occurrence, but a rule.

Here is another example illustrating the same phenomenon. For each $n \in \mathbb{N}$, let $T_{n}(t)$ and $B_{n}$ be the restrictions of $S_{n}(t)$ and $A_{n}$ given in (4.3) and (4.4) to $\mathbb{C}^{2}$, respectively, i.e.

$$
T_{n}(t)\left(\begin{array}{l}
x \\
y
\end{array}\right)=\left(\begin{array}{c}
\mathrm{e}^{-a_{n} t} x \\
\mathrm{e}^{-n t} y
\end{array}\right) \quad \text { and } \quad B_{n}\left(\begin{array}{l}
x \\
y
\end{array}\right)=\left(\begin{array}{c}
-a_{n} x \\
-n y
\end{array}\right) .
$$

Clearly, $\left(T_{n}\right)_{n \in \mathbb{N}}$ is a sequence of equibounded semigroups generated by the $B_{n}$ 's, with $X_{\text {almost-unif }}^{\mathrm{s}}=\mathbb{C} \times\{0\}$ and $X_{\text {point }}^{\mathrm{s}}=\mathbb{C} \times \mathbb{C}$. The $B_{n}$ 's also generate equibounded cosine families, 


$$
C_{n}(t)\left(\begin{array}{l}
x \\
y
\end{array}\right)=\left(\begin{array}{c}
\cos \left(\sqrt{a_{n}} t\right) x \\
\cos (\sqrt{n} t) y
\end{array}\right),
$$

but these, unlike the $T_{n}$ 's, converge merely on $X_{\text {almost-unif }}^{\mathrm{s}}\left(=X_{\text {point }}^{\mathrm{c}}=X_{\text {almost-unif }}^{\mathrm{c}}\right)$ which is the regularity space for the limit pseudo-resolvent corresponding to the resolvents of the $B_{n}$ 's. We remark that the disparity between $X_{\text {almost-unif }}^{\mathrm{s}}$ and $X_{\text {point }}^{\mathrm{s}}$ is related to the "smoothing property" of the Weierstrass formula: the semigroups derived from cosine families by means of this formula are much more "regular" than the cosine families themselves, and therefore, their trajectories typically converge for a larger sets of vectors than the trajectories of the cosine families.

We note that Theorem 3 gives information on individual trajectories as well: if, for some $x \in X, \lim _{n \rightarrow \infty} C_{n}(t) x$ exists for all $t \in \mathbb{R}$, then $x \in X_{\text {point }}^{\mathrm{c}}=X_{\text {almost-unif }}^{\mathrm{c}}$, and so the convergence is in fact almost uniform in $t \in \mathbb{R}$.

One noteworthy consequence of Theorem 3 is that-in contrast to the rich theory of singular perturbations of semigroups - the theory of singular perturbations of cosine families has no objects of study: there are no sequences of cosine families that converge in an irregular way.

We conclude this section with two supplementary results. The first of these asserts that, unlike in the case of semigroups (see e.g. [27]), there is only one cosine family, a trivial cosine family, for which the strong limit $\lim _{t \rightarrow \infty} C(t)$ exists. The proof relies on an argument involving a strongly convergent sequence of cosine families, hence the inclusion of the result in this section. The second result is a local version of the first result and asserts that if a trajectory of a bounded cosine family has limits at infinity, then it is constant. An immediate consequence of this last result is that the important theory of asymptotic behaviour of semigroups $[15,27]$ does not have a counterpart for cosine families.

We say that a cosine family $C=\{C(t)\}_{t \in \mathbb{R}}$ on a Banach space $X$ is trivial if $C(t)=$ $I_{X}$ for all $t \in \mathbb{R}$. If $C$ is strongly continuous, then $C$ is trivial if and only if the generator of $C$ is the zero operator.

PROPOSITION 2. Let $C=\{C(t)\}_{t \in \mathbb{R}}$ be a (non-necessarily strongly continuous) cosine family on a Banach space $X$ such that the limit

$$
P x:=\lim _{t \rightarrow \infty} C(t) x
$$

exists for all $x \in X$. Then $C$ is trivial and $P=I_{X}$.

Proof. For each $n \in \mathbb{N}$, let $C_{n}=\left\{C_{n}(t)\right\}_{t \in \mathbb{R}}$ be the cosine family defined by $C_{n}(t)=$ $C(n t)$. By assumption, $\left(C_{n}(t)\right)_{n \in \mathbb{N}}$ converges strongly to $P$ for $t>0$. Since $C(-t)=$ $C(t)$ for every $t \in \mathbb{R}$, it follows that $\left(C_{n}(t)\right)_{n \in \mathbb{N}}$ converges strongly to $P$ also for $t<0$. Since, in addition, $C_{n}(0)=I_{X}$ for all $n \in \mathbb{N}$, we see that $\left(C_{n}(t)\right)_{n \in \mathbb{N}}$ converges strongly for all $t \in \mathbb{R}$, and the limit $C_{\infty}(t)$ of $\left(C_{n}(t)\right)_{n \in \mathbb{N}}$ is equal to $P$ for $t \neq 0$ and to $I_{X}$ for $t=0$. Since, by the Banach-Steinhaus theorem, $\sup _{n \in \mathbb{N}}\left\|C_{n}(t)\right\|<\infty$ for every $t \in \mathbb{R}$ and since each $C_{n}$ satisfies the cosine functional equation, we immediately conclude that $C_{\infty}=\left\{C_{\infty}(t)\right\}_{t \in \mathbb{R}}$ satisfies the cosine functional equation. Now, if we 
fix $s \neq 0$ and substitute first $2 s$ for $t$ and next $s$ for $t$ in the formula $2 C_{\infty}(t) C_{\infty}(s)=$ $C_{\infty}(t+s)+C_{\infty}(t-s)$, then we get $2 P^{2}=2 P$ and $2 P^{2}=P+I_{X}$, respectively. This implies $P=I_{X}$. On the other hand, letting $s \rightarrow \infty$ in the formula $2 C(t) C(s) x=$ $C(t+s) x+C(t-s) x$, we obtain $2 C(t) x=2 C(t) P x=P x+P x=2 x$. Since this holds for all $t \in \mathbb{R}$ and all $x \in X$, the proposition is established.

We remark that if $C$ in the above proposition is assumed to be strongly continuous, then the equality $P=I_{X}$ can be established differently, in a much simpler way. Indeed, $C_{\infty}$ is then strongly measurable, being the strong limit of the strongly continuous families $C_{n}$, and hence, by Theorem $1, C_{\infty}$ is in fact strongly continuous. Now, $P=I_{X}$ is a consequence of the strong continuity of $C_{\infty}$ and the fact that $C_{\infty}(t)=P$ for $t \neq 0$ and $C_{\infty}(0)=I_{X}$.

The following corollary generalises [1, Proposition 3.14.6].

COROLLARY 1. Let $C=\{C(t)\}_{t \in \mathbb{R}}$ be a bounded cosine family on a Banach space $X$, and let $x \in X$ be such that the limit $\lim _{t \rightarrow \infty} C(t) x$ exists. Then $C(t) x=x$ for all $t \in \mathbb{R}$.

Proof. Let $X_{0}$ be composed of those elements $y$ of $X$ for which $\lim _{t \rightarrow \infty} C(t) y$ exists. By the boundedness of $C, X_{0}$ is a closed linear subspace of $X$. Exploiting the cosine functional equation, we readily deduce that each $C(t), t \in \mathbb{R}$, leaves $X_{0}$ invariant. Since the restriction $C_{\mid X_{0}}=\left\{C_{\mid X_{0}}(t)\right\}_{t \in \mathbb{R}}$ of $C$ to $X_{0}$ satisfies the assumptions of Proposition 2, it follows that $C(t)_{\mid X_{0}}=I_{X_{0}}$ for all $t \in \mathbb{R}$. As $x$ is in $X_{0}$, we have $C(t) x=x$ for all $t \in \mathbb{R}$, and the corollary is proved.

\section{Convergence may fail to be uniform}

We now turn our attention to another problem related to convergence of cosine families. As shown in [10], on $C_{0}(0, \infty]$, the cosine families related to elastic Brownian motions described in the previous section converge uniformly and not merely almost uniformly on $\mathbb{R}$. A similar (and more elementary) example of uniform convergence was described in [4]. Based on these results, it was conjectured that equibounded cosine families, if they converge, do so uniformly on $\mathbb{R}$. If true, this would expose yet another difference between convergence of semigroups and convergence of cosine families. However, the conjecture turns out to be false, and here we show that it fails with the aid of a certain general result and two specific examples of its use, and also by means of an independent result of a slightly different nature.

A continuous function $f$ on $\mathbb{R}$ with values in a Banach space $X$ is said to be (uniformly) almost periodic if the set of its translates $\left\{T_{t} f\right\}_{t \in \mathbb{R}}$ is relatively compact in the metric $\rho(f, g)=\sup _{t \in \mathbb{R}}\|f(t)-g(t)\|$; the translate $T_{t} f$ of $f$ by $t \in \mathbb{R}$ is given by the definition $T_{t} f(s)=f(t+s), s \in \mathbb{R}$. Let $A P(\mathbb{R}, X)$ be the space of all $X$-valued almost periodic functions on $\mathbb{R}$. For any $f \in A P(\mathbb{R}, X)$ and any $\alpha \in \mathbb{R}$, the mean value 


$$
M_{t}\left\{\mathrm{e}^{-i \alpha t} f(t)\right\}=\lim _{T \rightarrow \infty} \frac{1}{2 T} \int_{-T}^{T} \mathrm{e}^{-i \alpha t} f(t) \mathrm{d} t
$$

exists and defines the Fourier-Bohr coefficient of $f$ for the Fourier exponent $\alpha, \widehat{f}(\alpha)$. The Fourier-Bohr coefficients of $f$ vanish for all but at most countably many Fourier exponents. The set

$$
\Sigma(f)=\{\alpha \in \mathbb{R} \mid \widehat{f}(\alpha) \neq 0\}
$$

constitutes the Bohr spectrum of $f$ and is non-empty if $f$ is non-zero. The function $f$ is uniquely determined by its Fourier-Bohr coefficients, this property being implicitly meant when one refers to $f$ via its expansion into a formal Fourier-Bohr series

$$
f(t) \sim \sum_{\alpha \in \Sigma(f)} \mathrm{e}^{i \alpha t} \widehat{f}(\alpha) .
$$

For a function $f$ defined on $\mathbb{R}$ and $\lambda \in \mathbb{R}$, we denote by $f_{[\lambda]}$ the function on $\mathbb{R}$ given by

$$
f_{[\lambda]}(t)=f(\lambda t) \quad(x \in \mathbb{R}) .
$$

THEOREM 4. Let $f \in A P(\mathbb{R}, X)$ be non-constant, where $X$ is a Banach space, and let $\left(\lambda_{n}\right)_{n \in \mathbb{N}}$ be a sequence of real numbers converging to $\lambda \in \mathbb{R}$ and such that $\lambda_{n} \neq \lambda$ for each $n \in \mathbb{N}$. Then $\left(f_{\left[\lambda_{n}\right]}\right)_{n \in \mathbb{N}}$ converges to $f_{[\lambda]}$ almost uniformly but not uniformly on $\mathbb{R}$.

Proof. The almost uniform convergence of $\left(f_{\left[\lambda_{n}\right]}\right)_{n \in \mathbb{N}}$ to $f_{[\lambda]}$ stems from the fact that $f$ is uniformly continuous and the fact that the functions $t \mapsto \lambda_{n} t$ converge to $t \mapsto \lambda t$ almost uniformly on $\mathbb{R}$. All we then need is to show that the convergence of $\left(f_{\left[\lambda_{n}\right]}\right)_{n \in \mathbb{N}}$ is not uniform on $\mathbb{R}$.

Since $f$ is non-zero, $\Sigma(f)$ is non-empty. Let $\left(\alpha_{k}\right)_{k \in[K]}$ be an enumeration of $\Sigma(f)$, where $[K]$ denotes the interval of positive integers between 1 and $K$, with $K$ being finite or infinite. According to Bochner's theorem [12] (see also [14, Thm. 1.24]), there exists a sequence of trigonometric polynomials

$$
\sigma_{m}(t)=\sum_{k=1}^{K_{m}} r_{k, m} \mathrm{e}^{i \alpha_{k} t} \widehat{f}\left(\alpha_{k}\right) \quad(t \in \mathbb{R}, m \in \mathbb{N})
$$

which converges uniformly to $f$ on $\mathbb{R}$ in the norm topology of $X$, as $m \rightarrow \infty$, with $r_{k, m}$ being rational numbers that depend on $\alpha_{k}$ and $m$, but not on $\widehat{f}\left(\alpha_{k}\right)$, and satisfy

$$
\lim _{m \rightarrow \infty} r_{k, m}=1
$$

for every $k \in \mathbb{N}$. Let $\epsilon>0$ and let $k_{0}$ be an arbitrary member of $[K]$ such that $\alpha_{k_{0}} \neq 0$. Choose $m \in \mathbb{N}$ so that

$$
\left\|f(t)-\sigma_{m}(t)\right\|<\epsilon
$$

for each $t \in \mathbb{R}$ and

$$
r_{k_{0}, m} \geq \frac{1}{2}
$$


Suppose, on the contrary, that $\left(f_{\left[\lambda_{n}\right]}\right)_{n \in \mathbb{N}}$ converges to $f_{[\lambda]}$ uniformly on $\mathbb{R}$. Then, there exists $N_{0} \in \mathbb{N}$ such that if $n>N_{0}$, then

$$
\left\|f_{\left[\lambda_{n}\right]}(t)-f_{[\lambda]}(t)\right\|<\epsilon
$$

for each $t \in \mathbb{R}$. Consequently,

$$
\begin{aligned}
\left\|\sum_{k=1}^{K_{m}} r_{k, m}\left(\mathrm{e}^{i \alpha_{k} \lambda_{n} t}-\mathrm{e}^{i \alpha_{k} \lambda t}\right) \widehat{f}\left(\alpha_{k}\right)\right\| \leq & \left\|\sum_{k=1}^{K_{m}} r_{k, m} \mathrm{e}^{i \alpha_{k} \lambda_{n} t} \widehat{f}\left(\alpha_{k}\right)-f_{\left[\lambda_{n}\right]}(t)\right\| \\
& +\left\|\sum_{k=1}^{K_{m}} r_{k, m} \mathrm{e}^{i \alpha_{k} \lambda t} \widehat{f}\left(\alpha_{k}\right)-f_{[\lambda]}(t)\right\| \\
& +\left\|f_{\left[\lambda_{n}\right]}(t)-f_{[\lambda]}(t)\right\|<3 \epsilon
\end{aligned}
$$

for each $n>N_{0}$ and each $t \in \mathbb{R}$.

Note that, $\lambda_{n} \neq 0$ for all sufficiently large $n$ (i.e. for all $n>N$ for some $N$ ). Indeed, when $\lambda \neq 0$, this is obvious, and when $\lambda=0$, this follows from the assumption that $\lambda_{n} \neq \lambda$ for all $n$. Consequently, if $k \in\left\{1, \ldots, K_{m}\right\} \backslash\left\{k_{0}\right\}$, then

$$
\alpha_{k} \lambda_{n}-\alpha_{k_{0}} \lambda_{n} \neq 0
$$

for eventually all $n$.

Moreover, if $k \in\left\{1, \ldots, K_{m}\right\}$, then

$$
\alpha_{k} \lambda-\alpha_{k_{0}} \lambda_{n} \neq 0
$$

for eventually all $n$. For if $k \neq k_{0}$ and $\lambda \neq 0$, then this is clear since then $\lim _{n \rightarrow \infty} \alpha_{k} \lambda-$ $\alpha_{k_{0}} \lambda_{n}=\left(\alpha_{k}-\alpha_{k_{0}}\right) \lambda \neq 0$. If $\lambda=0$, then (5.4) reduces to $\alpha_{k_{0}} \lambda_{n} \neq 0$, and this holds because of $\alpha_{k_{0}} \neq 0$ and the assumption that $\lambda_{n} \neq \lambda$ for all $n$ which now reads $\lambda_{n} \neq 0$ for all $n$. Similarly, if $k=k_{0}$, then (5.4) holds by virtue of $\alpha_{k_{0}} \neq 0$ and $\lambda_{n} \neq \lambda$ for all $n$.

Let $N_{1}>N_{0}$ be such that (5.3) and (5.4) hold for $n>N_{1}$. Taking into account that

$$
M_{t}\left\{\mathrm{e}^{i \alpha t}\right\}= \begin{cases}1 & \text { if } \alpha=0 \\ 0 & \text { otherwise }\end{cases}
$$

we see that if $n>N_{1}$, then

$$
M_{t}\left\{\mathrm{e}^{i\left(\alpha_{k} \lambda_{n}-\alpha_{k_{0}} \lambda_{n}\right) t}\right\}=0
$$

whenever $k \in\left\{1, \ldots, K_{m}\right\} \backslash\left\{k_{0}\right\}$ and

$$
M_{t}\left\{\mathrm{e}^{i\left(\alpha_{k} \lambda-\alpha_{k_{0}} \lambda_{n}\right) t}\right\}=0
$$

whenever $k \in\left\{1, \ldots, K_{m}\right\}$. Consequently, fixing $n>N_{1}$ arbitrarily, we have

$$
M_{t}\left\{\mathrm{e}^{-i \alpha_{k_{0}} \lambda_{n} t}\left(\sum_{k=1}^{K_{m}} r_{k, m}\left(\mathrm{e}^{i \alpha_{k} \lambda_{n} t}-\mathrm{e}^{i \alpha_{k} \lambda t}\right) \widehat{f}\left(\alpha_{k}\right)\right)\right\}=r_{k_{0}, m} \widehat{f}\left(\alpha_{k_{0}}\right)
$$

On the other hand, by (5.2), 


$$
\left\|M_{t}\left\{\mathrm{e}^{-i \alpha_{k_{0}} \lambda_{n} t}\left(\sum_{k=1}^{K_{m}} r_{k, m}\left(\mathrm{e}^{i \alpha_{k} \lambda_{n} t}-\mathrm{e}^{i \alpha_{k} \lambda t}\right) \widehat{f}\left(\alpha_{k}\right)\right)\right\}\right\| \leq 3 \epsilon .
$$

Taking into account (5.1), we see that $\left\|\widehat{f}\left(\alpha_{k_{0}}\right)\right\| \leq 6 \epsilon$. Hence $\widehat{f}\left(\alpha_{k_{0}}\right)=0$, by the arbitrariness of $\epsilon$. We have thus shown that $\Sigma(f) \subset\{0\}$. This implies that $f$ is constant, a contradiction.

Let $C=\{C(t)\}_{t \in \mathbb{R}}$ be a strongly continuous cosine family on a Banach space $X$. For any $\lambda \in \mathbb{R}$, we denote by $C_{[\lambda]}$ the $X$-valued cosine family given by

$$
C_{[\lambda]}(t)=C(\lambda t) \quad(t \in \mathbb{R}) .
$$

An element $x \in X$ will be termed an almost periodic vector for $C$ if the $C$-trajectory associated with $x$ is almost periodic.

We note that if $\left(\lambda_{n}\right)_{n \in \mathbb{N}}$ is a sequence of real numbers converging to $\lambda \in \mathbb{R}$, then $\left(C_{\left[\lambda_{n}\right]}\right)_{n \in \mathbb{N}}$ converges to $C_{[\lambda]}$ strongly and almost uniformly in $t \in \mathbb{R}$. Indeed, arguing as in the proof of Theorem 4, this follows from the fact that, for each $x \in X, t \mapsto C(t) x$ is uniformly continuous on every compact subset of $\mathbb{R}$ and the fact that the functions $t \mapsto \lambda_{n} t$ converge to $t \mapsto \lambda t$ almost uniformly on $\mathbb{R}$.

An immediate consequence of Theorem 4 and the above observation is the following.

THEOREM 5. Let $C=\{C(t)\}_{t \in \mathbb{R}}$ be a strongly continuous cosine family on a Banach space $X$. Suppose that there exists an almost periodic vector for $C$ such that the $C$-trajectory associated with this vector is non-constant. Let $\left(\lambda_{n}\right)_{n \in \mathbb{N}}$ be a sequence of real numbers converging to $\lambda \in \mathbb{R}$ and such that $\lambda_{n} \neq \lambda$ for each $n \in \mathbb{N}$. Then $\left(C_{\left[\lambda_{n}\right]}\right)_{n \in \mathbb{N}}$ converges to $C_{[\lambda]}$ strongly and almost uniformly but not uniformly in $t \in \mathbb{R}$.

We now present two instances of use of the above result. In the first case, we let $X=\mathbb{C}$ and $C(t)=\cos t$ for each $t \in \mathbb{R}$ and further observe that $x=1$ is an almost periodic vector for $C$; then, an application of Theorem 5 leads to the following result:

EXAMPLE 1. If $\left(\lambda_{n}\right)_{n \in \mathbb{N}}$ is a sequence of real numbers converging to $\lambda \in \mathbb{R}$ and such that $\lambda_{n} \neq \lambda$ for each $n \in \mathbb{N}$, then the sequence $\left(\left\{\cos \lambda_{n} t\right\}_{t \in \mathbb{R}}\right)_{n \in \mathbb{N}}$ of the scalarvalued cosine families converges to the scalar-valued cosine family $\{\cos \lambda t\}_{t \in \mathbb{R}}$ almost uniformly but not uniformly on $\mathbb{R}$.

Before discussing the other case, we introduce a class $\mathscr{C}$ of cosine families as follows: a cosine family $C=\{C(t)\}_{t \in \mathbb{R}}$ on a Banach space $X$ is in $\mathscr{C}$ if only if the following conditions are satisfied:

(C1) there exists a sequence $\left(C_{n}\right)_{n \in \mathbb{N}}, C_{n}=\left\{C_{n}(t)\right\}_{t \in \mathbb{R}}$, of cosine families on $X$ converging to $C$ strongly and uniformly in $t \in \mathbb{R}$ and such that $C_{n} \neq C$ for every $n \in \mathbb{N}$;

(C2) there exists a sequence $\left(\tilde{C}_{n}\right)_{n \in \mathbb{N}}, \tilde{C}_{n}=\left\{\tilde{C}_{n}(t)\right\}_{t \in \mathbb{R}}$, of cosine families on $X$ converging to $C$ strongly and almost uniformly but not uniformly in $t \in \mathbb{R}$. 
With this definition in hand, we can now proceed to the other instance of use of Theorem 5. Based on Theorem 5, Example 2 below reveals that the class $\mathscr{C}$ is nonvoid. The significance of this result is that it demonstrates that the question of whether or not the limit of a sequence of cosine families is uniform or almost uniform cannot, in general, be answered in terms of the limit cosine family alone.

EXAMPLE 2. Let $c_{0}$ be the space of all complex sequences $\xi=\left(\xi_{k}\right)_{k \in \mathbb{N}}$ convergent to 0 , equipped with the usual supremum norm

$$
\|\xi\|_{\infty}=\sup _{k \in \mathbb{N}}\left|\xi_{k}\right|
$$

For each $n \in \mathbb{N}$, let $e_{n}$ be the element of $c_{0}$ such that

$$
\left(e_{n}\right)_{k}= \begin{cases}1 & \text { if } k=n \\ 0 & \text { otherwise }\end{cases}
$$

Every $\xi=\left(\xi_{k}\right)_{k \in \mathbb{N}}$ in $c_{0}$ can conveniently be represented as the series

$$
\xi=\sum_{k=1}^{\infty} \xi_{k} e_{k}
$$

convergent in the norm of $c_{0}$. For each $t \in \mathbb{R}$ and each $n \in \mathbb{N}$, let $C_{n}(t)$ be the linear operator on $c_{0}$ defined by

$$
C_{n}(t) \xi=\sum_{k=1}^{n}(\cos k t) \xi_{k} e_{k}+\sum_{k=n+1}^{\infty} \xi_{k} e_{k},
$$

and, for each $t \in \mathbb{R}$, let $C(t)$ be the linear operator on $c_{0}$ given by

$$
C(t) \xi=\sum_{k=1}^{\infty}(\cos k t) \xi_{k} e_{k}
$$

with $\xi=\left(\xi_{k}\right)_{k \in \mathbb{N}}$ being an arbitrary member of $c_{0}$. Clearly, for each $n \in \mathbb{N}, C_{n}=$ $\left\{C_{n}(t)\right\}_{t \in \mathbb{R}}$ is a strongly continuous cosine family of contractions on $c_{0}$, and also $C=\{C(t)\}_{t \in \mathbb{R}}$ is a strongly continuous cosine family of contractions on $c_{0}$. Given $\lambda \in \mathbb{R}$ and $n \in \mathbb{N}$, we shall write $C_{n,[\lambda]}$ in lieu of $\left(C_{n}\right)_{[\lambda]}$. For any $n \in \mathbb{N}$, any $t, \lambda \in \mathbb{R}$, and any $\xi \in c_{0}$, we have

$$
\begin{aligned}
\left\|C_{[\lambda]}(t) \xi-C_{n,[\lambda]}(t) \xi\right\|_{\infty} & \leq\left\|\sum_{k=n+1}^{\infty} \xi_{k} e_{k}\right\|\left\|_{\infty}+\right\| \sum_{k=n+1}^{\infty}(\cos \lambda k t) \xi_{k} e_{k} \|_{\infty} \\
& \leq \sup _{k>n}\left|\xi_{k}\right|+\sup _{k>n}\left|(\cos \lambda k t) \xi_{k}\right| \\
& \leq 2 \sup _{k>n}\left|\xi_{k}\right|,
\end{aligned}
$$

whence 


$$
\sup _{t \in \mathbb{R}}\left\|C_{[\lambda]}(t) \xi-C_{n,[\lambda]}(t) \xi\right\|_{\infty}=0 .
$$

Thus, for each $\lambda \in \mathbb{R}$, the sequence $\left(C_{n,[\lambda]}\right)_{n \in \mathbb{N}}$ converges to $C_{[\lambda]}$ strongly and uniformly on $\mathbb{R}$.

On the other hand, the representation (5.6) implies that every $\xi \in c_{0}$ is an almost periodic vector for $C$, and moreover, the function $t \mapsto C(t) \xi, t \in \mathbb{R}$, is non-constant whenever $\xi \neq 0$. Therefore, by Theorem 5 , if, for a given $\lambda \in \mathbb{R},\left(\lambda_{n}\right)_{n \in \mathbb{N}}$ is a sequence of real numbers converging to $\lambda \in \mathbb{R}$ and such that $\lambda_{n} \neq \lambda$ for each $n \in \mathbb{N}$, then the sequence $\left(C_{\left[\lambda_{n}\right]}\right)_{n \in \mathbb{N}}$ converges to $C_{[\lambda]}$ almost uniformly but not uniformly on $\mathbb{R}$ in the strong operator topology. Actually, invoking Theorem 4 directly, we can infer a bit more: for every non-zero $\xi$ in $c_{0}$ the functions $t \mapsto C_{\left[\lambda_{n}\right]}(t) \xi, n \in \mathbb{N}$, converge to $t \mapsto C_{[\lambda]}(t) \xi$ almost uniformly but not uniformly in $t \in \mathbb{R}$.

We thus see that for each $\lambda \in \mathbb{R}, C_{[\lambda]}$ is the limit of two sequences of cosine families, each comprising cosine families different from $C_{[\lambda]}$, such that one sequence converges uniformly, while the other converges almost uniformly but not uniformly on $\mathbb{R}$; in other words, $C_{[\lambda]}$ is a member of $\mathscr{C}$.

The final result of this section, namely Example 3 below, will show that for a cosine family $C$ to belong to $\mathscr{C}$ it is not necessary to possess an almost periodic vector for which the $C$-trajectory associated with this vector is non-constant. In particular, it will become clear that membership in $\mathscr{C}$ can be established by means other than Theorem 5 .

EXAMPLE 3. We consider $C_{0}(0, \infty)$ as being equipped with the uniform norm $\|f\|_{\infty}=\sup _{x \geq 0}|f(x)|$. Let $C=\{C(t)\}_{t \in \mathbb{R}}$ be the cosine family on $C_{0}(0, \infty]$ given by

$$
C(t) f(x)=\frac{1}{2}(f(x+t)+\tilde{f}(x-t)) \quad\left(f \in C_{0}(0, \infty], x \geq 0\right)
$$

for each $t \geq 0$, with $\tilde{f}$ being the odd extension of $f$ on the whole of $\mathbb{R}$. We claim that $C$ is a member of $\mathscr{C}$ and that $C$ has no non-zero almost periodic vector.

To prove the first assertion, let $F$ be a real even continuous function on $\mathbb{R}$ with a limit at infinity. Then, in particular, $F$ is bounded. For each $\epsilon \in \mathbb{R}$, let $M_{\epsilon F}$ be the operator of multiplication by $\mathrm{e}^{\epsilon F}$ on $C_{0}(0, \infty]$ :

$$
M_{\epsilon F} f=\mathrm{e}^{\epsilon F} f \quad\left(f \in C_{0}(0, \infty]\right) .
$$

Clearly, $M_{\epsilon F}$ is bounded, with $\left\|M_{\epsilon F}\right\| \leq \mathrm{e}^{|\epsilon|\|F\|_{\infty}}$, and the inverse of $M_{\epsilon F}$ coincides with $M_{-\epsilon F}$. For each $\epsilon \in \mathbb{R}$ and each $t \in \mathbb{R}$, set

$$
C_{(\epsilon)}(t)=M_{-\epsilon F} C(t) M_{\epsilon F}
$$

In view of the evenness of $F$, we have the following explicit expression

$$
\begin{gathered}
C_{(\epsilon)}(t) f(x)=\frac{1}{2}\left(f(x+t) \mathrm{e}^{\epsilon(F(x+t)-F(x))}+\tilde{f}(x-t) \mathrm{e}^{\epsilon(F(x-t)-F(x))}\right) \\
\left(f \in C_{0}(0, \infty], x \geq 0\right)
\end{gathered}
$$


for every $t \geq 0$. We remark that it is only for the purpose of obtaining this elegant formula that we assumed $F$ to be even - this assumption may be well dispensed with without detriment to the argument that follows. It is clear that for any $\epsilon \in \mathbb{R}, C_{(\epsilon)}(t)$ is a linear bounded operator on $C_{0}(0, \infty]$ with $\left\|C_{(\epsilon)}(t)\right\| \leq \mathrm{e}^{2|\epsilon|\|F\|_{\infty}}$ for every $t \in \mathbb{R}$, and $C_{(\epsilon)}=\left\{C_{(\epsilon)}(t)\right\}_{t \in \mathbb{R}}$ is a strongly continuous cosine family on $C_{0}(0, \infty)$. Let $\left(\epsilon_{n}\right)_{n \in \mathbb{N}}$ be a sequence of real numbers converging to 0 and let $\epsilon=\sup _{n \in \mathbb{N}}\left|\epsilon_{n}\right|$. Then, $\left(C_{\left(\epsilon_{n}\right)}\right)_{n \in \mathbb{N}}$ is a sequence of equibounded cosine families on $C_{0}(0, \infty)$, with $\left\|C_{\left(\epsilon_{n}\right)}(t)\right\| \leq \mathrm{e}^{2 \epsilon\|F\|_{\infty}}$ for each $n \in \mathbb{N}$ and each $t \in \mathbb{R}$. Since $\left(M_{-\epsilon_{n} F}\right)_{n \in \mathbb{N}}$ and $\left(M_{\epsilon_{n} F}\right)_{n \in \mathbb{N}}$ tend to the identity operator on $X$ in operator norm, we see that $\left(C_{\left(\epsilon_{n}\right)}\right)_{n \in \mathbb{N}}$ converges to $C$ in operator norm uniformly on $\mathbb{R}$. Thus, to finish the proof that $C$ is a member of $\mathscr{C}$, it suffices to find a sequence of cosine families on $C_{0}(0, \infty]$ that converges to $C$ strongly and almost uniformly but not uniformly on $\mathbb{R}$. We will show that for any sequence $\left(\lambda_{n}\right)_{n \in \mathbb{N}}$ of positive numbers converging to 1 and such that $\lambda_{n} \neq 1$ for each $n \in \mathbb{N},\left(C_{\left[\lambda_{n}\right]}\right)_{n \in \mathbb{N}}$ furnishes the desired sequence [we use the notation as per (5.5)].

As $\left(C_{\left[\lambda_{n}\right]}\right)_{n \in \mathbb{N}}$ converges to $C$ strongly and almost uniformly on $\mathbb{R}$, it suffices to show that the convergence is not uniform. We shall establish the following stronger statement: for any non-zero $f$ in $C_{0}(0, \infty]$, the functions $t \mapsto C_{\left[\lambda_{n}\right]}(t) f, n \in \mathbb{N}$, fail to converge uniformly to $t \mapsto C(t) f$. To this end, given $f \in C_{0}(0, \infty] \backslash\{0\}$, we first observe that for any $t \geq 0$ and $n \in \mathbb{N}$, the right-hand side of the formula

$$
\begin{aligned}
& C(t) f(x)-C_{\left[\lambda_{n}\right]}(t) f(x) \\
& \quad=\frac{1}{2}\left(f(x+t)-f\left(x+\lambda_{n} t\right)\right)+\frac{1}{2}\left(\tilde{f}(x-t)-\tilde{f}\left(x-\lambda_{n} t\right)\right) \quad(x \geq 0)
\end{aligned}
$$

reduces to

$$
\frac{1}{2}\left(f(2 t)-f\left(\left(1+\lambda_{n}\right) t\right)\right)-\frac{1}{2} \tilde{f}\left(\left(1-\lambda_{n}\right) t\right)
$$

if we let $x=t$. Thus, setting

$$
f_{n}(t)=\frac{1}{2}\left(f(2 t)-f\left(\left(1+\lambda_{n}\right) t\right)\right),
$$

we have

$$
\left\|C(t) f-C_{\left[\lambda_{n}\right]}(t) f\right\|_{\infty} \geq\left|f_{n}(t)-\frac{1}{2} \tilde{f}\left(\left(1-\lambda_{n}\right) t\right)\right| .
$$

As $\sup _{t \geq 0}\left|\tilde{f}\left(\left(1-\lambda_{n}\right) t\right)\right|=\|f\|_{\infty}$, our task will be complete once we show that $\lim _{n \rightarrow \infty}\left\|f_{n}\right\|_{\infty}=0$.

For any $\epsilon>0$, choose $T>0$ so that $|f(t)-f(\infty)|<\epsilon$ whenever $t>T$. Then, clearly, $\sup _{t>T}\left|f_{n}(t)\right| \leq \epsilon$ for all $n$. On the other hand, if $t \in[0, T]$, then $\left|2 t-\left(1+\lambda_{n}\right) t\right| \leq\left|1-\lambda_{n}\right| T$, and this in conjunction with the fact that $f$ is uniformly continuous on $[0, T]$ implies that $\sup _{t \in[0, T]}\left|f_{n}(t)\right| \leq \epsilon$ for all sufficiently large $n$. Thus, $\left\|f_{n}\right\|_{\infty} \leq \epsilon$ for all sufficiently large $n$, which finishes the proof that $C$ is in $\mathscr{C}$.

Passing to the proof of the second assertion, note that

$$
\lim _{t \rightarrow \infty} C(t) f(x)=\lim _{t \rightarrow-\infty} C(t) f(x)=0
$$


for any $f \in C_{0}(0, \infty]$ and any $x \geq 0$. Suppose now that $f \in C_{0}(0, \infty]$ is an almost periodic vector for $C$. Then, in view of the above equalities,

$$
\left[\lim _{T \rightarrow \infty} \frac{1}{2 T} \int_{-T}^{T} \mathrm{e}^{-i \alpha t} C(t) f \mathrm{~d} t\right](x)=\lim _{T \rightarrow \infty} \frac{1}{2 T} \int_{-T}^{T} \mathrm{e}^{-i \alpha t} C(t) f(x) \mathrm{d} t=0
$$

for each $\alpha \in \mathbb{R}$ and each $x \geq 0$. Thus, all the Fourier-Bohr coefficients of $t \mapsto C(t) f$ vanish and consequently $f=0$. The second assertion and the claim are established.

We conclude this section and the paper with two remarks. First, as it was already indicated at the beginning of this section, the operators $A_{n}$ specified in the paragraph following Theorem 3 generate cosine families $C_{n}$ on $C[0, \infty]$ that converge to $C$ on $C_{0}(0, \infty]$ strongly and uniformly in $t \in \mathbb{R}$ (see [10, Theorem 4.3]). Critically, the families $C_{n}$ do not have $C_{0}(0, \infty)$ for an invariant space, and hence, the restrictions of these families to $C_{0}(0, \infty)$ are not genuine cosine families on $C_{0}(0, \infty)$. This explains why a sequence of cosine families different from $\left(C_{n}\right)_{n \in \mathbb{N}}$, namely $\left(C_{\left(\epsilon_{n}\right)}\right)_{n \in \mathbb{N}}$, was used to prove that $C$ falls into $\mathscr{C}$ in the example above.

Second, we note that the same family $C$ considered as acting on the larger Banach space $B U C_{0}(0, \infty)$ of bounded, uniformly continuous functions on $[0, \infty)$ vanishing at 0 , belongs to $\mathscr{C}$, too. This can be directly deduced from Theorem 5 by observing that the sine function belongs to $B U C_{0}(0, \infty)$ and is an almost periodic vector (in fact a periodic vector, with the obvious meaning of the term) for $C$. While this result and that of Example 3 are similar in content, there is a difference between the arguments used for their establishment. The argument for the first result hinges on an application of Theorem 5 and proves the existence of a sequence of trajectories associated with a single vector for which the convergence is not uniform on $\mathbb{R}$. In contrast, the other argument, just as the argument underpinning Example 2, shows that for no non-zero vector, the corresponding trajectories converge.

\section{Acknowledgments}

This research was partially supported by the Polish Government under Grant 6081/B/H03/2011/40. The authors would like to thank S. Król for the reference to Lemma 5.2 in Fattorini's paper [16].

Open Access. This article is distributed under the terms of the Creative Commons Attribution License which permits any use, distribution, and reproduction in any medium, provided the original author(s) and the source are credited.

\section{REFERENCES}

[1] Arendt, W., Batty, C.J.K., Hieber, M., Neubrander, F.: Vector-Valued Laplace Transforms and Cauchy Problems. Birkhäuser, Basel (2001)

[2] Banasiak, J., Bobrowski, A.: Interplay between degenerate convergence of semigroups and asymptotic analysis: a study of a singularly perturbed abstract telegraph system. J. Evol. Equ. 9, 293-314 (2009) 
[3] Bobrowski, A.: Degenerate convergence of semigroups. Semigroup Forum 49, 303-327 (1994)

[4] Bobrowski, A.: The Widder-Arendt theorem on inverting of the Laplace transform, and its relationships with the theory of semigroups of operators. Methods Funct. Anal. Topology 3, 1-39 (1997)

[5] Bobrowski, A.: A note on convergence of semigroups. Ann. Polon. Math. 69, 107-127 (1998)

[6] Bobrowski, A.: Functional Analysis for Probability and Stochastic Processes. Cambridge University Press, Cambridge (2005)

[7] Bobrowski, A.: Degenerate convergence of semigroups related to a model of stochastic gene expression. Semigroup Forum 73, 345-366 (2006)

[8] Bobrowski, A.: On a semigroup generated by a convex combination of two Feller generators. J. Evol. Equ. 7, 555-565 (2007)

[9] Bobrowski, A.: On limitations and insufficiency of the Trotter-Kato theorem. Semigroup Forum 75, 317-336 (2007)

[10] Bobrowski, A.: Generation of cosine families via Lord Kelvin's method of images. J. Evol. Equ. 10, 663-675 (2010)

[11] Bobrowski, A.: From diffusions on graphs to Markov chains via asymptotic state lumping. Ann. Henri Poincaré 13, 1501-1510 (2012)

[12] Bochner, S.: Abstrakte Fastperiodische Funktionen. Acta Math. 61, 149-184 (1933)

[13] Chander, R., Singh, H.: On the measurability and continuity properties of the cosine operator. Indian J. Pure Appl. Math. 12, 81-83 (1981)

[14] Corduneanu, C.: Almost Periodic Functions. Interscience Publishers, New York (1968)

[15] Engel, K.J., Nagel, R.: One-Parameter Semigroups for Linear Evolution Equations. Springer, New York (2000)

[16] Fattorini, H.O.: Ordinary differential equations in linear topological spaces. I. J. Differential Equations 5, 72-105 (1969)

[17] Fattorini, H.O.: Ordinary differential equations in linear topological spaces. II. J. Differential Equations 6, 50-70 (1969)

[18] Fattorini, H.O.: Second order linear differential equations in Banach spaces. North-Holland, Amsterdam (1985)

[19] Goldstein, J.A.: On the convergence and approximation of cosine functions. Aequationes Math. 11, 201-205 (1974)

[20] Goldstein, J.A.: Semigroups of Linear Operators and Applications. Oxford University Press, New York (1985)

[21] Hille, E.: Une généralisation du problème de Cauchy. Ann. Inst. Fourier (Grenoble) 4, 31-48 (1952)

[22] Hille, E., Phillips, R.S.: Functional Analysis and Semi-Groups. Amer. Math. Soc. Colloq. Publ. 31. Amer. Math. Soc., Providence, R. I. (1957)

[23] Itô, K., McKean, Jr., H.P.: Diffusion Processes and Their Sample Paths. Springer, Berlin (1996). Repr. of the 1974 ed.

[24] Karatzas, I., Shreve, S.E.: Brownian Motion and Stochastic Calculus. Springer, New York (1991)

[25] Konishi, Y.: Cosine functions of operators in locally convex spaces. J. Fac. Sci. Univ. Tokyo Sect. IA Math. 18, 443-463 (1971/1972)

[26] Kurepa, S.: Semigroups and cosine functions. In: Functional Analysis (Dubrovnik, 1981), Lecture Notes in Math., vol. 948, pp. 47-72. Springer, Berlin (1982)

[27] Lasota, A., Mackey, M.C.: Chaos, Fractals, and Noise. Stochastic Aspects of Dynamics. Springer (1994)

[28] Piskarev, S., Shaw, S.Y.: On certain operator families related to cosine operator functions. Taiwanese J. Math. 1, 527-546 (1997)

[29] Vasil'ev, V.V., Piskarev, S.I.: Differential equations in Banach spaces. II. Theory of cosine operator functions. J. Math. Sci. (N. Y.) 122, 3055-3174 (2004) 


\section{A. Bobrowski}

Institute of Mathematics,

Polish Academy of Sciences,

Śniadeckich 8,00-956 Warsaw, Poland

E-mail: a.bobrowski@pollub.pl

W. Chojnacki

School of Computer Science,

The University of Adelaide,

Adelaide, SA 5005, Australia

and

Wydziat Matematyczno-Przyrodniczy,

Szkoła Nauk Ścistych,

Uniwersytet Kardynała Stefana Wyszyńskiego, Dewajtis 5, 01-815 Warsaw, Poland

E-mail: wojciech.chojnacki@adelaide.edu.au 